\title{
A importância do farmacêutico no tratamento da Diabetes mellitus tipo 2
}

\author{
The importance of the pharmacist in the treatment of type 2 Diabetes mellitus \\ La importancia del farmacéutico en el tratamiento de la Diabetes mellitus tipo 2
}

\section{Resumo}

Na busca por confirmar a importância do profissional farmacêutico no tratamento do DM, o objetivo deste artigo é identificar, através da literatura pertinente, a Atenção Farmacêutica prestada ao paciente portador de Diabetes Mellitus tipo 2. Trata-se de uma Revisão Integrativa da Literatura do tipo qualitativa, utilizando as bases de dados: Scielo, Pubmed e Lilacs. A Diabetes Mellitus tipo 2 é uma doença metabólica que vem crescendo muito nos últimos tempos devido ao estilo de vida sedentário que as pessoas aderem, além de uma alimentação inadequada, esse grupo populacional precisa utilizar hipoglicemiantes orais ou insulina para manter dentro da normalidade os níveis glicêmicos. O farmacêutico é o profissional capacitado para orientar quanto ao tratamento medicamentoso e até mesmo o não medicamentoso, todos os cuidados com a interação de drogas, administração ou armazenamento de insulina devem ser explicados ao paciente para que o mesmo possa realizar o tratamento com eficácia. Assim, conclui-se que a Atenção Farmacêutica é indispensável para orientar e conscientizar o portador de DM, atuando em conjunto com a equipe multidisciplinar.

Palavras-chave: Diabetes mellitus tipo 2; Assistência farmacêutica; Tratamento medicamentoso.

\begin{abstract}
In the search to confirm the importance of the pharmacist in the treatment of DM, the aim of this article is to identify, through the relevant literature, the Pharmaceutical Care provided to patients with type 2 Diabetes Mellitus. This is an Integrative Literature Review of type 2. qualitative, using the databases: Scielo, Pubmed and Lilacs. Type 2 Diabetes Mellitus is a metabolic disease that has been growing a lot in recent times due to the sedentary lifestyle that people adhere to, in addition to an inadequate diet, this population group needs to use oral hypoglycemic agents or insulin to maintain blood glucose levels within normal limits. The pharmacist is the professional trained to advise on drug treatment and even non-drug treatment, all precautions with drug interaction, insulin administration or storage must be explained to the patient so that he can perform the treatment effectively. Thus, it is concluded that Pharmaceutical Care is essential to guide and raise awareness of DM patients, working together with the multidisciplinary team.
\end{abstract}

Keywords: Type 2 Diabetes mellitus; Pharmaceutical care; Drug treatment.

\section{Resumen}

En la búsqueda por confirmar la importancia del farmacéutico en el tratamiento de la DM, el objetivo de este artículo es identificar, a través de la literatura relevante, la Atención Farmacéutica que se brinda a los pacientes con Diabetes Mellitus tipo 2. Se trata de una Revisión Literaria Integrativa de tipo 2. cualitativo, utilizando las bases de datos: Scielo, Pubmed y Lilacs. La Diabetes Mellitus tipo 2 es una enfermedad metabólica que ha ido creciendo mucho en los últimos tiempos debido al sedentarismo al que se adhieren las personas, además de una dieta inadecuada, este grupo de población necesita utilizar hipoglucemiantes orales o insulina para mantener los niveles de glucosa en sangre. dentro de los límites normales. El farmacéutico es el profesional capacitado para asesorar sobre el tratamiento farmacológico e incluso el tratamiento no farmacológico, todas las precauciones con la interacción farmacológica, la administración o el almacenamiento de insulina deben ser explicadas al paciente para que pueda realizar el tratamiento de forma eficaz. Así, se concluye que la Atención Farmacéutica es fundamental para orientar y sensibilizar a los pacientes con DM, trabajando en conjunto con el equipo multidisciplinar.

Palabras clave: Diabetes mellitus tipo 2; Cuidado farmacéutico; Tratamiento farmacológico. 


\section{Introdução}

As doenças crônicas não transmissíveis (DCNT) são um dos maiores problemas e desafios da saúde púbica, visto que se tem um elevado número de óbitos, além de diminuir gradativamente a qualidade das pessoas acometidas, incapacitando-as de realizar as atividades do cotidiano com eficácia. Essas comorbidades apresentam também um alto custo para o sistema público de saúde, pois requer acompanhamento regular com a equipe multiprofissional e tratamento contínuo, seja medicamentoso ou não medicamentoso (Lima et al., 2018).

As principais doenças crônicas que acometem o indivíduo no Brasil são: Hipertensão Arterial (HA) e Diabetes Mellitus (DM), seguido por Doença renal Crônica (DRC). O envelhecimento da população brasileira, concomitante a obesidade e estilo de vida sedentária dentro do processo de urbanização em que se tem uma sociedade globalizada e preocupada apenas com as atividades do dia a dia no trabalho, deixando sempre em segundo plano a promoção da saúde, são as principais causas dessas doenças (Flor; Campos, 2017).

A DM é uma doença considerada crônica e progressiva, em que o indivíduo acometido tem seu estado de saúde deteriorado com o passar dos tempos, pois surgem complicações inerentes ao descontrole glicêmico, com impacto físico e psicológico significativos. Por isso o diagnóstico dessa comorbidade deve ser fechado o mais precoce possível para que o tratamento seja iniciado e os valores glicêmicos voltem à normalidade (Sinclair et al., 2017).

A dextrose, conhecida como glicose, consiste num monossacarídeo, uma molécula simples de carboidrato que tem a importante função de fornecer energia para as células, ajudando no seu funcionamento. Todo alimento que contém carboidrato (massas, pães), possui glicose na sua composição e por isso essa molécula está presente na alimentação do dia a dia, a maior parte é composto por três monossacarídeos: glicose, frutose e galactose. O açúcar ou sacarose consiste na junção de dois monossacarídeos (glicose e frutose), já o leite ou lactose é composto de um monossacarídeo (galactose). A frutose e a galactose precisam ser transformadas em glicose no fígado para que as células possam utilizar como fonte de energia (Brasil, 2020).

A glicose é a única molécula de carboidrato que pode fornecer energia no funcionamento das células de todo ser vivo. Fisiologicamente, após cada refeição os carboidratos ingeridos passam pelo processo de digestão, onde é quebrado em vários micro pedaços, libertando os monossacarídeos, mas é no intestino delgado que essas moléculas são absorvidas e vão para a corrente sanguínea, aumentando a glicemia (aumento dos níveis de glicose no sangue). Para compensar, as células beta do pâncreas produzem um hormônio chamado insulina que é liberado quando a glicemia está elevada, o qual faz com que a glicose presente na corrente sanguínea entre nas células do corpo. Esse hormônio é fundamental para a homeostase, visto que também tem a função de armazenar glicose no fígado para que em algum momento de necessidade o organismo tenha uma fonte de glicose, independente da alimentação (Brasil, 2020).

Compreende-se então que a Diabetes consiste numa morbidade que o organismo possui grande dificuldade para controlar os níveis de glicose no sangue, o motivo pode ser a produção insuficiente ou ausente de insulina no pâncreas ou pode ser a incapacidade das células de reconhecer esse hormônio, não deixando a glicose entrar, e nas duas formas há um acúmulo da glicemia (Assunção et al., 2017).

Sabendo do impacto que a Diabetes Mellitus tipo 2 causa na qualidade de vida das pessoas, o problema da pesquisa baseia-se nos desafios que o farmacêutico enfrenta para conscientizar quanto a adesão do tratamento, assim como o uso racional dos medicamentos. Sendo assim, o presente estudo contribuirá para a academia na medida que trata sobre a assistência farmacêutica no tratamento de pessoas com DM tipo 2, envolvendo desde a dispensação dos medicamentos até a orientação e conscientização para uso correto dos fármacos.

A DM tipo 2 tem como principal problema a resistência que o corpo adquire a insulina produzida, o que impede as células de captar a glicose circulante na corrente sanguínea. Esse tipo específico acontece devido ao estilo de vida que a pessoa 
segue, pois acomete em sua maioria pessoas obesas e sedentárias, muitas vezes associado ao histórico familiar para a doença. Principalmente pessoas com obesidade e acúmulo excessivo de gordura abdominal possuem maior risco de se tornar diabéticas, o que normalmente vem acompanhado de outra patologia como a Hipertensão Arterial ou Colesterol elevado, essa é uma condição clínica conhecida como síndrome metabólica, sendo um fator de risco importante para doenças cardiovasculares (Brasil, 2020).

São outros fatores de risco para a DM tipo 2: tabagismo, dieta rica em gorduras saturadas e carboidrato (pobre em vegetais e frutas), glicemia de jejum maior de $100 \mathrm{mg} / \mathrm{dl}$, história prévia de Diabetes Gestacional, idade entre 30 e 59 anos, ovário policístico, uso prolongado de medicamentos como corticoide. Já os sinais e sintomas são principalmente: polifagia, polidipsia, poliúria e fadiga (Costa et al., 2017).

O tratamento da DM tipo 2 deve ser realizado com mudanças no estilo de vida como prática de exercícios físicos e dieta regular, mas também deve ser realizado através do uso de medicamentos como os hipoglicemiantes orais. A adesão ao tratamento da DM tem sido um grande desafio para a equipe multidisciplinar, devido às mudanças que ocorrem na vida do indivíduo que não são fáceis de serem colocadas em prática. É muito comum que o paciente queira apenas tomar a medicação, mas sabe-se que os efeitos são melhores quando combinado com uma alimentação saudável e prática de atividade física regular, além disso, algumas vezes o paciente não faz uso racional das medicações e algo feito para ajudar pode causar complicações à saúde, por isso se faz necessário o acompanhamento multiprofissional (Pereira; Frizon, 2017).

A Atenção Farmacêutica é um termo criado na década de 80, trazendo a ideia de que as necessidades farmacoterápicas de um indivíduo não diz respeito somente a dispensação de medicação, mas também os serviços que ofereçam uma farmacoterapia segura, com uso racional dos fármacos, garantindo a eficácia. Com o passar dos tempos, o farmacêutico ampliou sua área de atuação, tornando-se responsável por utilizar seu conhecimento, flexibilidade e habilidade na melhor abordagem para o tratamento farmacológico da Diabetes (Rolim et al., 2016).

As práticas farmacêuticas agregam um conjunto de atitudes, valores éticos, comportamentos, habilidades e responsabilidades, utilizadas na prevenção de doenças, promoção da saúde e recuperação do indivíduo. Além disso, o farmacêutico deve traçar meios educativos em saúde a respeito da importância do uso correto e consciente das medicações pelos pacientes. Portanto, o cuidado farmacêutico está diretamente ligado à prática da farmacoterapia que objetive melhorar a qualidade de vida e principalmente promover saúde ao paciente (Nicoletti; Kubota, 2017).

A atenção farmacêutica voltada ao paciente portador de Diabetes Mellitus é indispensável pelo fato de que essa morbidade é uma das que mais afeta a população no Brasil e no mundo, e essas pessoas estão sujeitas Problemas Relacionados a Medicamentos (PRMs) pela falta de orientação relacionada ao uso consciente e racional das medicações, as quais precisam ser tomadas continuamente, assim como a maior parte dessas pessoas não possuem conhecimento adequado das complicações causadas, do controle da doença e das interações medicamentosas e alimentares que interferem na ação da medicação (Silva; Souza, 2017; Brentegani; Zampieron, 2017).

As atividades assistenciais do farmacêutico estão contidas na Atenção Farmacêutica, que procura orientar o paciente com relação ao medicamento utilizado no acompanhamento farmacoterapêutico. Dentro da equipe multiprofissional, o farmacêutico é o profissional mais habilitado para orientar o usuário, com base na sua formação voltada a farmacodinâmica e farmacocinética dos fármacos, desempenhando uma assistência de qualidade e eficiente. Esse profissional precisa garantir que o usuário entendeu corretamente o uso das medicações para que possa cumprir os esquemas farmacoterápicos e alcançar resultados favoráveis no tratamento e controle da glicemia (Saturno et al., 2016).

Para que a assistência seja realizada com base científica o farmacêutico precisa estar sempre procurando especializarse e atualizar-se sobre as medicações existentes no tratamento da DM tipo 2, mas além dos conhecimentos técnicos esse 
profissional precisa trabalhar de forma humanizada escutando as queixas do paciente, retirando suas dúvidas e entendendo o mesmo diante da sua realidade de vida (Souza; Garcia, 2019).

Algumas pessoas portadoras da DM tipo 2 necessitam fazer uso de insulina, as mais utilizadas são NPH e Regular, no momento da dispensação da insulina exógena, o usuário precisa de orientações sobre a administração e armazenamento. Por isso, a Atenção Farmacêutica é imprescindível para que a terapêutica seja mantida buscando o objetivo principal que consiste em fazer o controle glicêmico. Para aquele paciente que foi prescrito hipoglicemiante oral, também é necessário orientações a respeito do horário e dosagem certa. A adesão farmacológica também é um problema enfrentado pelo farmacêutico, sendo necessário conscientizar o usuário sobre a importância de realizar o tratamento adequado para que não haja complicações associadas a ausência da terapia medicamentosa (Silva et al., 2016).

Mas, além disso, o farmacêutico precisa orientar o cliente a respeito do tratamento não farmacológico que consiste na reeducação alimentar e prática regular de atividades físicas, deixando claro que é preciso unir as duas formas terapêuticas para que o tratamento tenha eficácia e o indivíduo volte a ter qualidade de vida. Dessa forma, entende-se que esse profissional é fundamental para o acompanhamento terapêutico do paciente com DM tipo 2, desde a dispensação de medicamentos até orientações diversas (Campos et al., 2020).

Na busca por confirmar a importância do profissional farmacêutico no tratamento do DM, o objetivo deste artigo é identificar, através da literatura pertinente, a Atenção Farmacêutica prestada ao paciente portador de Diabetes Mellitus tipo 2.

\section{Metodologia}

Trata-se de uma Revisão da Literatura, do tipo Integrativa, seguindo o método qualitativo. Este tipo de pesquisa permite resumir os conhecimentos encontrados, assim como permite a análise crítica dos resultados relevantes de cada estudo, sendo importante dar prioridade a literatura mais recente para um melhor embasamento teórico científico sobre o tema proposto (Casarin et al., 2020).

Por se tratar de uma Revisão de Literatura, foi necessário utilizar as bases de dados eletrônicas: Scientific Electronic Library Online (SCIELO), PUBMED e Literatura Latino-americana e do Caribe em Ciências da Saúde (LILACS). Para a pesquisa, utilizou-se os seguintes descritores: Diabetes Mellitus tipo 2; Assistência Farmacêutica; Tratamento Medicamentoso, cruzando com os operadores booleanos “AND” e "OR'.

Utilizaram-se métodos primários para a coleta de dados, buscando informações científicas que sejam autênticas e objetivas, utilizando métodos qualitativos.

Os Critérios de Inclusão estabelecidos foram: artigos que tratem sobre o tema proposto, publicados entre os anos de 2016 a 2021, nos idiomas português e inglês. Critérios de Exclusão: Artigos indexados em mais de uma base de dados, artigos não disponíveis na íntegra dentro das bases de dados escolhidas e comentários não científicos.

Todos os artigos encontrados foram lidos na íntegra para a seleção daqueles que se encaixam nos critérios de inclusão. Os artigos foram organizados e analisados categorizando-os conforme os dados coletados na literatura, tendo como base a pesquisa qualitativa para facilitar a compreensão dos dados.

Não será necessário submeter o presente estudo ao Comitê de Ética em Pesquisa (CEP), tendo em vista que o mesmo não consiste numa pesquisa com seres humanos, sendo assim não oferecerá riscos físicos, químicos, biológicos ou psicológicos aos envolvidos. 


\section{Resultados e Discussão}

Segue abaixo para elucidação e melhor entendimento da temática abordada, um quadro mostrando os resultados preliminares da pesquisa de revisão de literatura, onde foram analisados e incluídos um total de 20 artigos, obedecendo os critérios de inclusão e exclusão já estabelecidos.

Quadro 1 - Detalhamento dos estudos encontrados, pré-selecionados, excluídos e incluídos.

\begin{tabular}{|c|c|c|c|c|}
\hline Base de dados & Encontrados & Pré-selecionados & Excluídos & Incluídos \\
\hline LILACS & 38 & 21 & 16 & 05 \\
\hline SCIELO & 93 & 56 & 44 & 12 \\
\hline PUBMED & 44 & 14 & 11 & 03 \\
\hline Total & $\mathbf{1 7 5}$ & $\mathbf{9 1}$ & $\mathbf{7 1}$ & $\mathbf{2 0}$ \\
\hline
\end{tabular}

Fonte: Elaboração própria.

Silva e Souza (2017) realizaram uma pesquisa no Pará com 30 pacientes utilizando a metodologia Dáder para acompanhamento farmacoterapêutico, os quais eram portadores de Diabetes tipo 2 e atendidos em uma unidade de saúde localizada em Santarém. Neste estudo destaca-se que o farmacêutico, nessa cidade, está ausente no Sistema Único de Saúde, acarretando em falhas na farmacoterapia. Assim que foi aplicado a atenção farmacêutica, observou-se que houve uma melhora significativa no tratamento dos pacientes que estavam sendo acompanhados pelos médicos, enfermeiros e demais profissionais da equipe de saúde.

Além dos inúmeros desafios que o farmacêutico precisa enfrentar para garantir que o paciente tenha um tratamento adequado para a doença metabólica, ainda é possível se deparar com situações em que este profissional não está incluso na terapêutica de uma forma geral, quando sabe-se a importância do mesmo na atuação com toda a equipe multidisciplinar.

Concomitante a isso, a pesquisa de Merlini e colaboradores (2019), acompanhou 25 voluntários funcionários de uma empresa de São Paulo que possuem o diagnóstico de Diabetes Mellitus tipo 1 e 2, ou Diabetes Gestacional, identificando que a comorbidade mais associada a diabetes foi a Hipertensão Arterial, além de que esses indivíduos apresentavam idade entre 34 e 76 anos e $52 \%$ eram obesos grau I. Os autores conseguiram identificar que a maioria dessas pessoas consomem medicamentos sem orientação médica ou farmacêutica, e o medicamento mais usado para controle glicêmico é a metformina, além da insulina. A maior parte desses voluntários fazem interações medicamentosas e apresentam problemas relacionados a medicamentos, demonstrando mais uma vez que a atenção farmacêutica é extremamente importante e necessária para a promoção da saúde da pessoa com Diabetes Mellitus.

Sabendo que nos dias atuais a DM é considerada uma epidemia em curso no mundo graças ao crescimento e envelhecimento da população, e principalmente com relação direta com alimentação inadequada e sedentarismo, os estudos concordam quando enfatizam a imprescindibilidade do farmacêutico no tratamento de doenças como o Diabetes Mellitus tipo 2, já que o objetivo do tratamento é manter os valores glicêmicos dentro da normalidade, evitando possíveis lesões em órgãos importantes, reduzindo também os riscos de complicações vasculares, renais, oculares e neurológicos, a partir do uso de hipoglicemiantes orais ou insulinas (Alencar et al., 2017; Campos et al., 2020; Marlini et al., 2019).

Essa terapêutica exige que o paciente entenda sobre os efeitos colaterais da medicação, sobre as consequências de escolher não aderir ao tratamento medicamentoso e também exige que o indivíduo tenha conhecimento sobre a importância da mudança do seu estilo de vida. Essas são ações da atenção farmacêutica, onde esse profissional torna-se responsável pela educação em saúde do paciente, assim como pela conscientização do mesmo para adesão correta da terapia. 
$\mathrm{Na}$ atenção farmacêutica, o paciente sempre será o mais importante, por isso esse profissional adquire, em sua formação, a capacidade de ter uma visão holística sobre o contexto geral, isso quer dizer que o mesmo deve ter um conjunto de comportamentos, atitudes, funções, conhecimentos e habilidades na assistência com a farmacoterapia, tendo como principal objetivo alcançar resultados terapêuticos positivos que contribuam para a melhora da qualidade de vida do indivíduo (Alencar et al., 2017).

Um estudo realizado por Gomes e colaboradores (2021), fez uma pesquisa com cem pessoas através da coleta de dados sociodemográficos, clínicos e laboratoriais. Dentre os medicamentos mais usados por esse grupo populacional, que foi em sua maioria do sexo feminino, destacou-se a metformina em monoterapia e a metformina associada as sulfoniluréias, além da insulina associada a um hipoglicemiante oral. Observou-se neste estudo que os pacientes tiveram um bom controle glicêmico em uso da metformina associada ou não com outro antidiabético. Mas também destacou que nos últimos tempos o aumento de pessoas com prescrição de medicamentos para esta doença metabólica se tornou significativo, o que implica na necessidade de um acompanhamento farmacoterapêutico ainda mais eficaz.

Alguns estudos apontam que a prevalência maior da Diabetes Mellitus é nas pessoas da terceira idade, dessa forma, entende-se que a função do farmacêutico é de extrema importância também nesse grupo prioritário, através da orientação sobre os medicamentos utilizados pelos idosos, já que a maior parte deles possuem pouco ou nenhum esclarecimento e compreensão sobre a utilização dos fármacos, interações medicamentosas e efeitos colaterais. É necessário enfatizar que o farmacêutico deve estar inserido na equipe multidisciplinar para orientação adequada da terapia medicamentosa e não medicamentosa em qualquer faixa etária (Pedroso; Staine, 2019; Gomes et al., 2021).

A importância da Atenção Farmacêutica ao diabético insulinodependente é ainda mais evidenciada, visto que é necessário um guia de orientações sobre o armazenamento, dosagem e aplicação da insulina, assim como é importante orientar sobre o descarte dos insumos utilizados na aplicação. Segundo Souza e Garcia (2019) o farmacêutico consegue identificar os diabéticos portadores de complicações crônicas para uma orientação mais rigorosa de prevenção dos agravos, também consegue construir junto ao paciente um mecanismo de rodízio dos locais de aplicação da insulina. Esses autores identificaram que quando não existe uma Atenção Farmacêutica no acompanhamento deste paciente, é muito comum práticas erradas de aplicação da insulina ou erros no local de armazenamento, o que reflete no descontrole glicêmico do indivíduo.

Por isso que a atuação do farmacêutico na equipe multiprofissional é indispensável para agregar conhecimento, promovendo ações que melhorem os hábitos de vida da população diabética, de maneira que reduza a automedicação e acima de tudo melhore a qualidade de vida do paciente.

\section{Conclusão}

O estudo dos artigos selecionados mostrou que o farmacêutico é um profissional imprescindível na equipe multidisciplinar para as orientações na terapêutica dos pacientes com Diabetes Mellitus tipo 2, já que essas pessoas precisam fazer uso de medicamentos, em monoterapia ou politerapia, ou ainda de insulina, que exige um conhecimento e aprendizado ainda mais efetivo sobre como utilizar, armazenar e descartar. Dentre as ações deste profissional, está a orientação quanto ao uso das medicações e sua interação com outros fármacos, assim como a explicação de seus efeitos colaterais e o uso racional desses medicamentos.

Assim, a Atenção farmacêutica é necessária em todos os níveis da saúde, atuando em conjunto com toda a equipe para construir uma visão holística do paciente e sua realidade. O farmacêutico contribui positivamente na construção de uma população consciente, orientada e informada sobre seu estado de saúde e sobre o seu tratamento medicamentoso ou não medicamentoso. 
Por fim, esta pesquisa possui uma relevância social para que haja o fortalecimento do profissional farmacêutico no tratamento de pessoas com DM. Os dados obtidos poderão embasar pesquisas futuras que estejam dentro do tema proposto de maneira que aumente o apoio que esse profissional precisa para mostrar sua capacidade teórica e prática de atuar junto a equipe multidisciplinar na terapêutica do paciente com diabetes, bem como em todos os níveis de saúde.

\section{Agradecimentos}

Agradecemos primeiramente a Deus, que em sua infinita bondade nos concedeu saúde, e força para juntas vencermos esta etapa de nossas vidas. Às nossas mães, que sempre foram nossa maior fonte de inspiração e força. Aos nossos irmãos, nossas famílias, e amigos, por sempre acreditarem em nossos sonhos, e entenderem nossos momentos de ausência. Aos nossos professores que contribuíram na nossa trajetória acadêmica, em especial ao professor João Paulo de Melo Guedes, responsável pela orientação desse trabalho, obrigada por compartilhar conosco sua sabedoria, o seu tempo e paciência. Por fim, a todos que contribuíram para que esse nosso sonho, se tornasse realidade. Nossa eterna gratidão.

\section{Referências}

Alencar, F. D. L. R. B., Costa, A. J. S., Neto, F. G. C., Dantas, J. C., \& Neto, E. M. R. (2017). Cuidados farmacêuticos ao paciente portador de diabetes mellitus: Revisão de literatura. Centro universitário católico de Quixadá.

Assunção, S. C., Fonseca, A. P., Silveira, M. F., Caldeira, A. P., \& Pinho, L. (2017). Conhecimento e atitude de pacientes com diabetes mellitus da atenção primária à saúde. Escola Anna Nery.

Brasil. (2020). Diretrizes sociedade brasileira de diabetes 2019-2020. Clannad editora científica.

Brentegani, K. R., \& Zampieron, F. G. (2017) A importância da atenção farmacêutica para portadores de diabetes mellitus tipo dois em drogarias: Uma revisão bibliográfica. Instituto de ciências da saúde.

Campos, L. S., Silva, C. B., Wanderley, T. L. R., Candeia, V. M. M., \& Calzerra, N. T. M. (2020) A prática da atenção farmacêutica no acompanhamento farmacoterapêutico de idosos diabéticos e hipertensos: Relato de caso. Braz. J. Hea. Rev. 3(2), 2287-2296.

Casarin, S. T., Porto, A. R., Gabatz, R. I. B., Bonow, C. A., \& Ribeiro, J. P.; Mota, M. S. (2020). Tipos de revisão de literatura: Considerações das editoras do Journal of Nursing and Health. J. nurs.

Costa, A. F., Flor, L. S., Campos, M. R., Oliveira, A. F., \& Costa, M. F. S. (2017). Carga do diabetes mellitus tipo 2 no Brasil. Cadernos de Saúde pública.

Flor, L. S., \& Campos, M. R. (2017) Prevalência de diabetes mellitus e fatores associados na população adulta brasileira: Evidências de um inquérito de base populacional. Revista Brasileira de Epidemiologia.

Gomes, G. J., Sartori, G. L., Sampaio, A. M., Pereira, L. M. V., Ueta, J. M., \& Oliveira, R. E. M. (2021). Tratamento e controle do diabetes mellitus tipo 2 em unidades de saúde da família de um município. Revista saúde em redes.

Lima, L. R., Fungheto, S. S., Volpe, C. R. G., Santos, W. S., Funez, M. I., \& Stival, M. M. (2018) Qualidade de vida e o tempo do diagnóstico do diabetes mellitus em idosos. Revista Brasileira de Geriatria e Gerontologia.

Merlini, V.A., Fernandes, F.B., \& Fernandes, S.M.S. (2019). Implementação de atenção farmacêutica como ferramenta para prevenção e acompanhamento do diabetes mellitus. Trabalho de Conclusão de Curso.

Nicoletti, M. A., \& Kubota, L. T. (2017). Benefícios decorrentes de prática do cuidado farmacêutico em hipertensão e diabetes tipo 2 para sua efetivação em unidades de saúde. 29. A Infarma ciências farmacêuticas.

Pereira, J., \& Frizon, E. (2017). Adesão ao tratamento nutricional de portadores de diabetes mellitus tipo 2: Uma revisão bibliográfica. [Manuscrito publicado, Revista da Associação Brasileira de Nutrição].

Pedroso, L. S., \& Staine, A. B. (2019). Caracterização do perfil de pacientes portadores de diabetes mellitus tipo-2 em uma unidade básica de saúde no município de Laranjeiras do Sul/PR. Revista Polidisciplinar Eletrônica.

Rolim, C. E., Rosa, S. P. S., Dias, J. M. F., Goncalves, S. A. A., \& Medeiros, A. P. (2016). A importância da atenção farmacêutica e a diabetes mellitus tipo 2. 10(2), 92. INTESA - Informativo Técnico do Semiárido.

Saturno, R. S., Santos, S. L. F., Rafael, E. S., Alves, H. H. S., \& Arraes, M. L. B. M. (2016). Estudo de caso: serviço de atenção farmacêutica ao idoso com diabetes tipo II. Mostra Científica da Farmácia. Centro Universitário Católica de Quixadá.

Silva, C., \& Souza, J. (2017). O farmacêutico na unidade básica de saúde: Atenção farmacêutica ao portador de Diabetes mellitus em uma unidade de saúde pública, no município de Santarém/PA. Acta Farmacêutica Portuguesa. 
Research, Society and Development, v. 10, n. 14, e573101422352, 2021

(CC BY 4.0) | ISSN 2525-3409 | DOI: http://dx.doi.org/10.33448/rsd-v10i14.22352

Silva, A. B., Engroff, P., Sgnaolin, V., Ely, L. S., \& Gomes, I. (2016). Prevalência de diabetes mellitus e adesão medicamentosa em idosos da Estratégia Saúde da Família de Porto Alegre/RS. Cadernos de saúde coletiva.

Sinclair, A. J., Abdelhafiz, A., Dunning, T., \& Izquierdo, M. (2017). An international position statement on the management of frailty in diabetes mellitus: summary of recommendations 2017. Springer link.

Souza, A. F., \& Garcia, R. M. A. (2019). A importância da atenção farmacêutica para o acompanhamento do paciente portador de diabetes insulinodependente. (2a ad.). Revista Científica Eletrônica. 